\title{
Histomorphometric Evaluation of the Small Coronary Arteries in Rats Exposed to Industrial Noise
}

\author{
Ana Lousinha ${ }^{1, *}$, Eduardo Antunes ${ }^{1}$, Gonçalo Borrecho ${ }^{1}$, Maria João Oliveira ${ }^{2}$, José Brito ${ }^{1}$ \\ and José Martins dos Santos ${ }^{1}$
}

1 Center for Interdisciplinary Research Egas Moniz (CIIEM), Health Sciences Institute, 2829-511 Monte de Caparica, Portugal; E-Mails: ejpantunes@sapo.pt (E.A.); gborrecho@gmail.com (G.B.); jaabrito@netcabo.pt (J.B.); jsantos@egasmoniz.edu.pt (J.M.S.)

2 Department of Anatomy, Abel Salazar Biomedical Sciences Institute, University of Porto, 4050-313 Porto, Portugal; E-Mail: mjoliveira@icbas.up.pt

* Author to whom correspondence should be addressed; E-Mail: alousinha@gmail.com; Tel.: +351-963-137-972.

Academic Editor: Francis J. Miller, Jr.

Received: 30 January 2015 / Accepted: 14 April 2015 / Published: 4 May 2015

\begin{abstract}
Morphological changes induced by industrial noise (IN) have been experimentally observed in several organs. Histological observations of the coronary arteries showed prominent perivascular tissue and fibrosis among IN-exposed rats. The effects on the small arteries are unknown. Objective: To evaluate the histomorphometric changes induced by IN on rat heart small arteries. Methods: Twenty Wistar rats exposed to IN during a maximum period of seven months and 20 age-matched controls were studied. Hearts were transversely sectioned from ventricular apex to atria and a mid-ventricular fragment was selected for analysis. The histological images were obtained with an optical microscope using 400× magnifications. A total of 634 arterial vessels (298 IN-exposed and 336 controls) were selected. The mean lumen-to-vessel wall $(\mathrm{L} / \mathrm{W})$ and mean vessel wall-to-perivascular tissue (W/P) ratios were calculated using image J software. Results: There were no differences between exposed and control animals in their L/W ratios $(p=0.687)$ and time variations in this ratio were non-significant $(p=0.110)$. In contrast, exposed animals showed lower $\mathrm{W} / \mathrm{P}$ ratios than control animals $(p<0.001)$, with significant time variations $(p=0.004)$. Conclusions: Industrial noise induced an increase in the perivascular tissue of rat small coronary arteries, with significant development of periarterial fibrosis.
\end{abstract}


Keywords: industrial noise; small coronary arteries; low-frequency noise

\section{Introduction}

Industrial noise (IN) is characterized by high intensity and a wide spectrum of wavelengths that includes low-frequency noise (LFN), this last characterized by large pressure amplitude $\geq 90 \mathrm{~dB}$ and low-frequency bands of $\leq 500 \mathrm{~Hz}$ [1]. Several morphological changes induced by IN and LFN have been experimentally observed in several tissues and organs [1-7].

We previously reported that coronary artery vessels showed prominent perivascular tissue and fibrotic development among IN-exposed rats [2] and also a significant fibrotic development in ventricular myocardium of rats exposed to LFN [3]. Considering the epidemiological evidence relating noise to ischemic heart disease and hypertension [8] and the effects of LFN on the extracellular matrix between the cardiomyocytes and around the cardiac vessels, which ultimately lead to myocardial stiffness and left ventricular disfunction and possibly to cardiac heart failure and arrhythmias $[9,10]$, additional studies were performed. These showed a reduction of cardiac connexin 43 and a significant increase of cardiac collagen I and III after LFN exposure [4,5], reinforcing the hypothesis of an inducible morphological arrhythmogenic substrate.

The effects on the morphology of small arteries and arterioles in the rat heart are currently unknown, namely to what extent arteriolar wall thickening and perivascular fibrosis of the heart can be influenced by IN and LFN.

The aim of this study was to characterize the structural changes induced by IN on the rat heart small arteries.

\section{Results}

\subsection{Data in Totum}

\subsubsection{Lumen-to-Vessel Wall Ratio}

We found no differences in the lumen-to-vessel wall $(\mathrm{L} / \mathrm{W})$ ratio between exposed and non-exposed animals. The histological evaluation did not show the presence of inflammatory cells in the two groups or modifications in the lumen or in the vessel wall.

The results of the histomorphometric analysis are shown in Table 1 . The mean L/W ratio was 0.5560 and 0.5619 , respectively, in groups A and B.

The two-way ANOVA analysis of the data showed no differences between exposed and control animals in their $\mathrm{L} / \mathrm{W}$ ratios $(p=0.687)$ and the time variations in this ratio were not significant $(p=0.110)$, as shown in Figure 1 .

However, since a significant interaction between the independent variables was found in the model ( $p=0.046$ ), with observed power of $64.9 \%$, we performed multiple comparisons by means of planned contrasts at different times that showed that, at month 7 , there were significant differences between groups for this ratio in totum, with significantly decreased values in exposed animals compared to controls $(p=0.034)$. 
Regarding differences between the groups in what concerns the time variations in this ratio, such differences were significant only for the changes observed from month 1 to $7(p=0.007)$, expressed as a $15.3 \%$ increase in the control group versus a $6.2 \%$ decrease in the exposed animals.

Table 1. Lumen-to-vessel wall ratio descriptive statistics in each group and overall, at the different times ( $\mathrm{IN}=$ industrial noise).

\begin{tabular}{ccccc}
\hline Group & Exposure Time (Months) & Mean & Standard Deviation & $\boldsymbol{N}$ \\
\hline & 1 & 0.5828933 & 0.03119288 & 5 \\
IN exposed (group A) & 3 & 0.5579890 & 0.02053823 & 5 \\
& 5 & 0.5361110 & 0.04120586 & 5 \\
& 7 & 0.5471695 & 0.04270947 & 5 \\
& Total & 0.5560407 & 0.03675745 & 20 \\
\hline \multirow{3}{*}{ Control (group B) } & 1 & 0.5301545 & 0.04229613 & 5 \\
& 3 & 0.5820185 & 0.06370201 & 5 \\
& 5 & 0.5242906 & 0.06810608 & 5 \\
& 7 & 0.6111611 & 0.03569630 & 5 \\
& Total & 0.5619062 & 0.06211472 & 20 \\
\hline
\end{tabular}

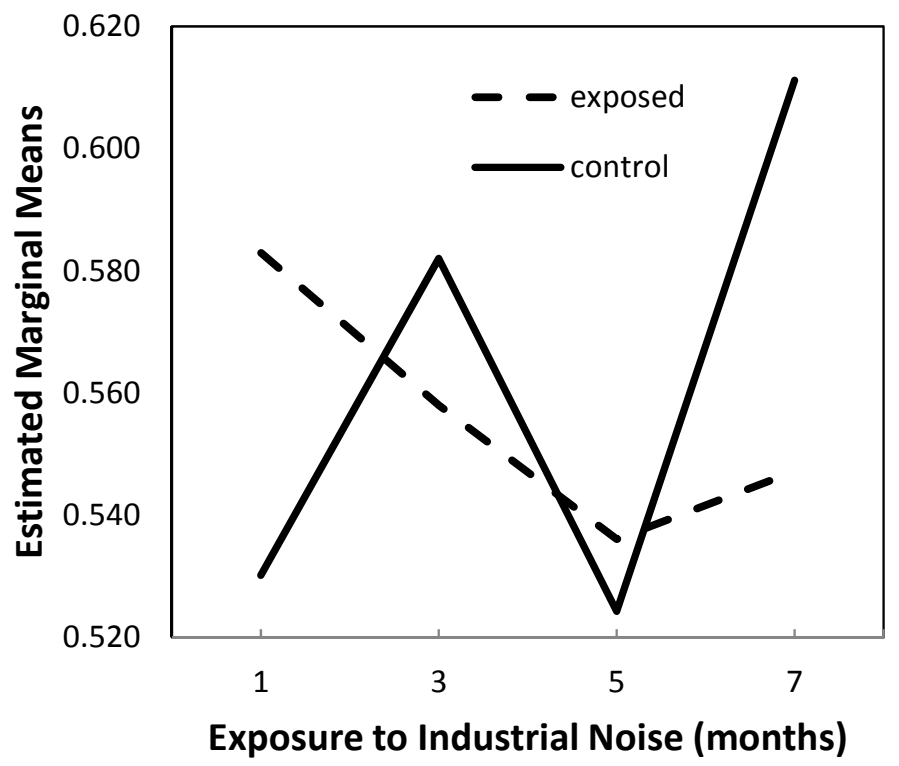

Figure 1. Estimated marginal means of lumen-to-vessel wall ratio.

\subsubsection{Wall-to-Perivascular Tissue Ratio}

By contrast, the perivascular tissue was more prominent and seemed to show fibrosis in IN-exposed rats. Sections of the arteries are shown in Figure 2. 


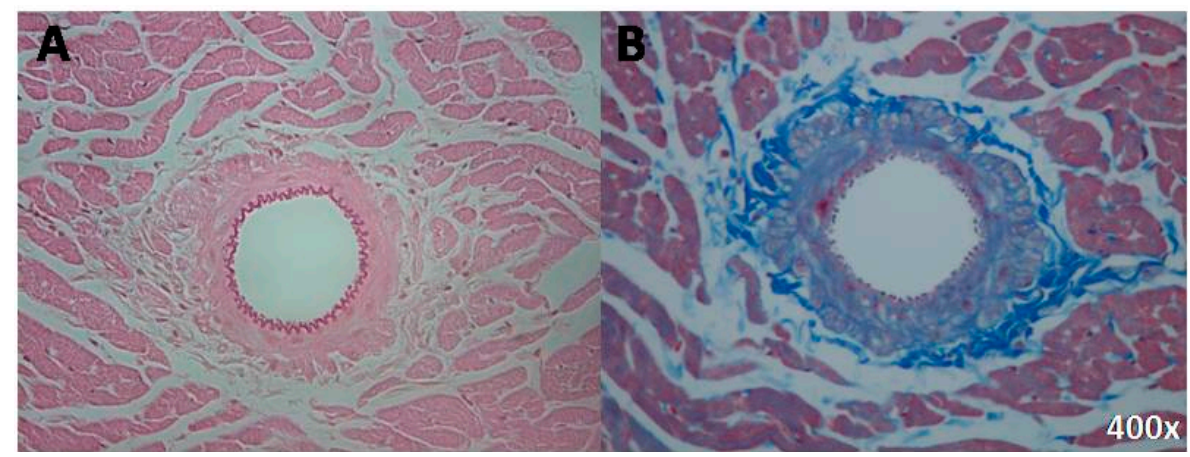

Figure 2. (A) Small coronary artery with a prominent perivascular tissue (IN group) (Hematoxylin-Eosin, 400×); (B) Small coronary artery with a prominent perivascular tissue, in the same group (Chromotrope-Aniline blue, 400×).

The mean wall-to-perivascular tissue (W/P) ratio was 0.4209 and 0.6373 , respectively, in groups A and B (Table 2).

Table 2. Wall-to-perivascular tissue ratio descriptive statistics in each group and overall, at the different times $(\mathrm{IN}=$ industrial noise $)$.

\begin{tabular}{ccccc}
\hline Group & Exposure Time (Months) & Mean & Standard Deviation & $\boldsymbol{N}$ \\
\hline & 1 & 0.4322535 & 0.03050318 & 5 \\
IN exposed (group A) & 3 & 0.3986722 & 0.02655070 & 5 \\
& 5 & 0.4234890 & 0.05262512 & 5 \\
& 7 & 0.4292097 & 0.04201934 & 5 \\
& Total & 0.4209061 & 0.03850858 & 20 \\
\hline \multirow{3}{*}{ Control (group B) } & 1 & 0.5849600 & 0.04896260 & 5 \\
& 3 & 0.6778928 & 0.01880381 & 5 \\
& 5 & 0.5745753 & 0.07750708 & 5 \\
& 7 & 0.7117764 & 0.01747083 & 5 \\
& Total & 0.6373011 & 0.07454984 & 20 \\
\hline
\end{tabular}

The results of the two-way ANOVA showed that there were significant effects on W/P ratio due to exposure $(p<0.001)$ and time variations $(p=0.004)$, which, however, are secondary, since a significant interaction between exposure and time exists $(p=0.001)$, as shown in Figure 3.

It is important to note that these effects do not seem to be due to chance, as the observed power is in excess of $90.7 \%$.

In view of this significant interaction, planned contrasts were applied in the post hoc comparisons, which allow the following conclusions:

(1) At months 1, 3, 5 and 7, exposed animals have significantly lower $\mathrm{W} / \mathrm{P}$ ratio than control animals $(p<0.001)$;

(2) From month 1 to 3, there is a 7.8\% decrease in W/P ratio in exposed animals which differs significantly $(p=0.003$ ) from the $15.9 \%$ increase in the same ratio observed in the control animals;

(3) From month 3 to 5 , there is a $6.0 \%$ increase in $\mathrm{W} / \mathrm{P}$ ratio in exposed animals, which differs significantly $(p=0.002)$ from the $15.2 \%$ decrease observed in the control animals; 
(4) From month 5 to 7 , there is an increase of $1.4 \%$ in W/P ratio in exposed animals, which is significantly lower $(p=0.002)$ than that of $23.8 \%$ observed in control animals.

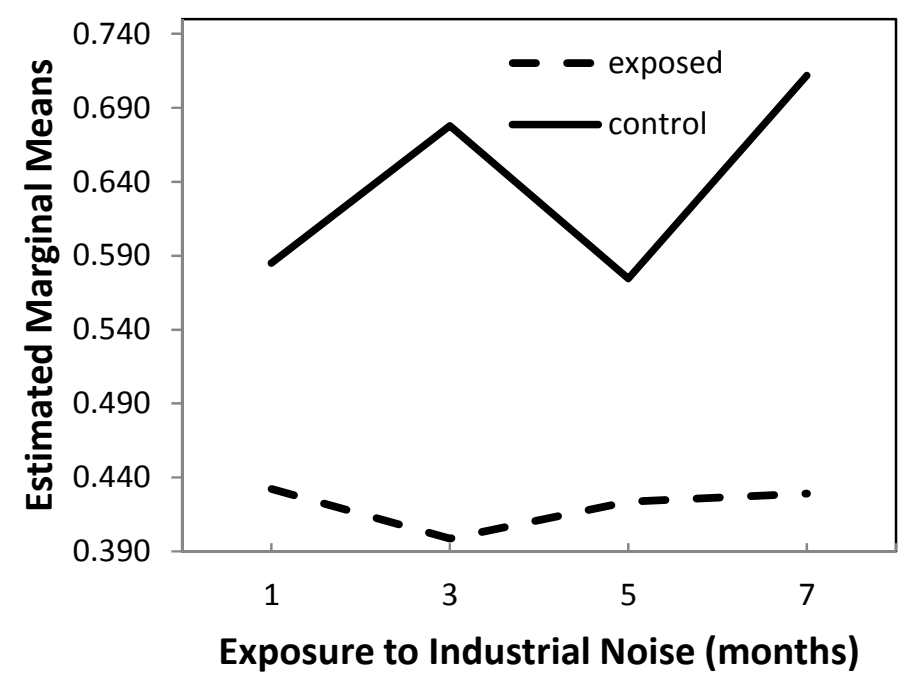

Figure 3. Estimated marginal means of wall-to-perivascular tissue ratio.

\subsection{Data Per Anatomic Region}

\subsubsection{Lumen-to-Vessel Wall Ratio}

The analysis of the data has shown that there were no significant effects of exposure to noise, duration of exposure and interaction between such factors on the $\mathrm{L} / \mathrm{W}$ ratio in any the anatomic regions considered: right ventricle (RV), septum and left ventricle (LV).

\subsubsection{Wall-to-Perivascular Tissue Ratio}

\section{Right Ventricle}

Significantly decreased W/P ratios were observed in the RV of exposed animal comparatively to controls $(p>0.001)$. No effect of duration $(p=0.111)$ or interaction between exposure and duration $(p=0.208)$ were observed in the same anatomic region.

\section{Septum}

The Two-way ANOVA approach to the data shows that there were significant effects on the W/P ratio in septum due to exposure $(p<0.001)$ and duration $(p=0.006)$, which were secondary, because of the significant interaction between exposure and duration $(p=0.002)$. It is important to note that these effects did not seem to be due to chance, as the observed power was in excess of $87.7 \%$.

In view of the significant interaction, planned contrasts were applied in the post hoc comparisons, with the following conclusions:

(1) Exposed animals had significantly lower $\mathrm{W} / \mathrm{P}$ ratio in septum than control animals, at all times $(p \leq 0.006)$; 
(2) From month 1 to 3, there was a $13.8 \%$ decrease in W/P ratio in exposed animals which differed significantly ( $p=0.003$ ) from the $18.8 \%$ increase in the same ratio observed in the control animals;

(3) From month 3 to 5, there was a 13.2\% increase in W/P ratio in exposed animals which differed significantly $(p=0.004)$ from the $16.7 \%$ decrease observed in the control animals;

(4) From month 5 to 7, there was an increase of 3.1\% in W/P ratio in exposed animals which was significantly lower $(p=0.006)$ than that of $30.6 \%$ observed in control animals.

\section{Left Ventricle}

The Two-way ANOVA showed that there were significant effects on the W/P ratio in the LV due to exposure $(p<0.001)$ but not to duration $(p=0.282)$, and a significant interaction between exposure and duration was found $(p<0.001)$. Again, these effects did not seem to be due to chance, as the observed power was in excess of $98.3 \%$.

Planned contrasts were applied in the post hoc comparisons, with the following conclusions:

(1) Exposed animals had significantly lower W/P ratio in LV than control animals, at all times $(p<0.001)$;

(2) From month 1 to 3, there was a 9.9\% decrease in W/P ratio in exposed animals which differed significantly $(p=0.010)$ from the $12.9 \%$ increase in the same ratio observed in the control animals;

(3) From month 3 to 5, there was a $14.0 \%$ increase in W/P ratio in exposed animals which differed significantly $(p=0.006)$ from the $10.8 \%$ decrease observed in the control animals;

(4) From month 5 to 7, there was a decrease of $12.3 \%$ in W/P ratio in exposed animals which was significantly different $(p=0.006)$ from the increase of $20.3 \%$ observed in control animals.

\section{Discussion}

A number of animal studies found modifications in several tissues induced by low-frequency noise, characterized by abnormal deposition of collagen in the extracellular matrices [1-7].

In the present study we found an increase in the perivascular tissue around the small coronary arteries in rats exposed to IN. This is in agreement with the previous findings of our group, concerning the histomorphometric evaluation of the coronary arterial vessels in rats exposed to industrial noise [3]. In both studies we found the development of perivascular fibrosis in the absence of inflammatory cells and in the absence of obstructive coronary artery disease.

There were significant differences between exposed rats and controls concerning the mean vessel wall-to-perivascular tissue ratio, higher among the control group $(p<0.001)$. The effects of exposure time were observed in the whole population of rats and seemed to be independent of exposure to IN. The perivascular tissue was more exuberant at three months of IN exposure, with a gradual reduction observed until seven months. It is important to note that, despite this gradual reduction, it remained increased among the IN-exposed rats compared to control at all times.

The analysis of data per anatomic region showed no particular differences between the right ventricle, septum and left ventricle, confirming that the influence of industrial noise over the heart is global.

Regarding the results concerning the ratio lumen-to-vessel wall between exposed and non-exposed animals, no significant differences were found until seven months of exposure to IN. At this point, 
an increased wall thickeness of intramyocardial small coronary arteries was observed in exposed rats, as compared to controls $(p=0.034)$. Previous studies performed on large vessels found the same type of modifications in the vessel wall [6]. In contrast, such differences were not observed in the coronary arterial vessels [3]. In this case, we speculated that a lower susceptibility of coronary arterial vessels to IN damage may occur, as suggested by the absence of the internal elastic lamina disruption and the fact that no proliferation of smooth muscle cells was observed in the intima up to seven months of exposure. Taking into consideration our recent results, we may further speculate that industrial noise effects on wall thickening require longer times of exposure and that small coronary arteries are the first to be affected.

A previous review of epidemiological studies concerning environmental noise exposure (including road and aircraft noise sources) and cardiovascular risk reported increasing evidence relating noise and hypertension and ischemic heart disease [8], making pertinent to investigate the effects of IN on the heart.

Thus far, we documented that coronary artery vessels showed prominent perivascular tissue and fibrotic development among IN-exposed rats [3] and also a significant fibrotic development in ventricular myocardium of rats exposed to LFN [2]. Considering that these structural changes ultimately lead to myocardial stiffness and left ventricular disfunction and possibly to cardiac heart failure and arrhythmias [9,10], additional studies were performed. These showed a reduction of cardiac connexin 43 and a significant increase of cardiac collagen I and III after LFN exposure [4,5], reinforcing the hypothesis of an inducible morphological arrhythmogenic substrate. The present study allowed the documentation of structural changes induced by industrial noise on the rat heart small arteries, suggesting a general influence of industrial noise over the heart.

It remains to understand the fibrotic proliferation mechanism behind the IN. A strong possibility, taking into consideration the dynamic interactions between fibroblasts and the extracellular matrix [11], could be the occurrence of an abnormal biological fibroblastic response induced by IN through a mecanotransduction process [12]. In addition, we could speculate that IN can induce fibrosis through the loss of regulation between profibrotic and antifibrotic molecules, carried out by mechanical and neurohumoral factors [13].

The changes in the structure of coronary resistance vessels, with quantitative characterization of small coronary arteries and arterioles in the myocardium, have been extensively studied under several experimental conditions and were already extended to humans. Cardiac hypertrophy in hypertension, with an increase in left ventricular mass, is characterised by increased wall thickness in arterioles and small arteries, increased lumen to wall ratio, and decreased number of capillary profiles per arteriole in cross section [14]. Volume overload-induced cardiac hypertrophy is characterized by normal coronary reserve and maximal flow and there is evidence that both arteriolar and capillary growth is proportional to the magnitude of hypertrophy [15]. Pathologic findings have described diabetic cardiomyopathy as a microvascular disease and previous studies have demonstrated that structural changes of coronary microvessels in diabetes include thickening of the vascular wall, perivascular fibrosis, capillary aneurysms, and decrease in capillary density [16,17]. Several structural abnormalities of the heart are present in chronic renal failure, including arteriolar thickening [18], reduced capillary density [19], and interstitial fibrosis [20]. These findings contribute to myocardial ischemia, left ventricular wall stiffness, diastolic dysfunction, and arrhythmogenicity in patients with renal failure [21]. 
It will be our challenge in the future to understand the clinical impact on cardiac diseases among humans exposed to industrial and low-frequency noise.

We are aware of several limitations of the study. We admit that interpretation of the results must be done cautiously because, for logistical reasons, the number of animals per group was limited. Additionally, small coronary arteries in biopsy samples were partially crushed during the procedure, which might have affected the quantitative analyses of lumen area and perivascular tissue. Furthermore, we acknowledge that at the present time there is not a well-defined morphological cardiac model induced by industrial noise. Thus far, we have limited our observations to the structural modifications induced by industrial noise or by low-frequency noise in the myocardium of rat heart and can only extrapolate these observations to humans, with all the existing limitations. The experimental conditions tried to simulate the schedule of industrial plant workers, characterized by 8 hours/day, 5 days/week of exposure to industrial noise. Once again, we reinforce the need of clinical investigations concerning the effects of industrial and low-frequency noise on the heart.

\section{Experimental Section}

Forty adult Wistar rats were studied. The animals were treated in accordance with the EU Commission on Animal Protection for Experimental and Scientific Purposes (86/609/EEC) and with the Portuguese legislation for the same purpose (Decree-Law No. 197/96). All the animals were kept in cages, fed standard rat food, and had free access to water. Twenty animals (Group A) were exposed to IN for a period of one to seven months, in an occupationally simulated schedule ( 8 hours/day, 5 days/week, and weekends in silence). The remaining 20 rats were used as age-matched controls (Group B) and were kept in a silent environment. Each group was divided into four subgroups with five rats and sacrificed after 1, 3, 5 and 7 months.

The sound signal was emitted by an analog noise generator, amplified and frequency filtered. The noise level was the same as previously reported, characterized by a wide spectrum of frequencies but with an important component under $500 \mathrm{~Hz}$ [11].

The hearts were fixed in $10 \%$ buffered formalin, sectioned transversely from the ventricular apex to the atria and prepared for histological observation using hematoxylin eosin and chromotrope-aniline blue (CAB) staining. The mid-ventricular fragment from each heart was selected for the study. The histological images were acquired with an optical microscope using $400 \times$ magnification.

A total of 634 arterial vessels were selected (298 in group A and 336 in group B). Data were analyzed using the computer image analysis image $\mathrm{J}$ software (National Institutes of Health, Bethesda, MA, USA). The caliber of the arterial vessels, the thickness of the walls and the perivascular tissue dimension were measured and the mean lumen-to-vessel wall $(\mathrm{L} / \mathrm{W})$ and mean vessel wall-to-perivascular tissue $(\mathrm{W} / \mathrm{P})$ ratios were calculated.

\section{Statistical Analysis}

The morphometric data are presented as mean \pm standard deviation. A two-way ANOVA model was applied in order to compare animals exposed to noise with non-exposed age-matched controls, in what concerns time variations (at months $1,3,5$ and 7) in the $\mathrm{L} / \mathrm{W}$ and $\mathrm{W} / \mathrm{P}$ ratios assessed in the cardiac muscle, in totum and per anatomic region (right ventricle, septum, and left ventricle). The 
appropriateness of the ANOVA model is justified by the fact that the 8 subgroups defined by the factors levels are of equal size $(n=5)$, which warrants the robustness of the F ratio statistics under potential heterogeneous variances. A $p$ value $<0.05$ was considered statistically significant.

\section{Conclusions}

In conclusion, industrial noise induced an increase in the perivascular tissue of rat small coronary arteries, with significant development of periarterial fibrosis.

\section{Author Contributions}

Ana Lousinha conceived and designed the experiments, performed the histomorphometric evaluation, analyzed the data and wrote the article; Eduardo Antunes conceived and designed the experiments, performed the histomorphometric evaluation and participated in the revision of manuscript content; Gonçalo Borrecho performed the experiments and participated in the histomorphometric evaluation; Maria João Oliveira conceived the experiments; José Brito performed the statistical analysis; José Martins dos Santos participated in the revision of manuscript content. All authors read and approved the final manuscript.

\section{Conflicts of Interest}

The authors declare no conflict of interest.

\section{References}

1. Oliveira, M.J.R.; Monteiro, M.; Ribeiro, A.M.; Pignatelli, D.; Aguas, A.P. Chronic exposure of rats to occupational textile noise cause cytological changes in adrenal cortex. Noise Health 2009, 11, 118-123.

2. Antunes, E.; Oliveira, P.; Borrecho, G.; Oliveira, M.J.R.; Brito, J.; Águas, A.; Martins dos Santos, J. Myocardial fibrosis in rats exposed to low-frequency noise. Acta Cardiol. 2013, 68, 241-245.

3. Antunes, E.; Oliveira, P.; Oliveira, M.J.R.; Brito, J.; Águas, A.; Martins dos Santos, J. Histomorphometric evaluation of the coronary artery vessels in rats submitted to industrial noise. Acta Cardiol. 2013, 68, 285-289.

4. Antunes, E.; Borrecho, G.; Oliveira, P.; Brito, J.; Águas, A.; Martins dos Santos, J. Immunohistochemical evaluation of cardiac connexin43 in rats exposed to low-frequency noise. Int. J. Clin. Exp. Pathol. 2013, 6, 1874-1879.

5. Antunes, E.; Borrecho, G.; Oliveira, P.; Matos, A.; Brito, J.; Águas, A.; Martins dos Santos, J. Effects of low-frequency noise on cardiac collagen and cardiomyocyte ultrastructure: An immunohistochemical and electron microscopy study. Int. J. Clin. Exp. Pathol. 2013, 6, 2333-2341.

6. Martins dos Santos, J.; Grande, N.R.; Castelo Branco, N.A.; Zagalo, C.; Oliveira, P. Vascular lesions and vibroacoustic disease. Eur. J. Anat. 2002, 6, 17-21.

7. Oliveira, P.; Brito, J.; Mendes, J.; da Fonseca, J.; Águas, A.; Martins dos Santos, J. Effects of large pressure amplitude low frequency noise in the parotid gland perivascular-ductal connective tissue. Acta Med. Port. 2013, 26, 237-242. 
8. Babish, W. Transportation noise and cardiovascular risk: updated review and synthesis of epidemiological studies indicate that the evidence has increased. Noise Health 2009, 8, 1-29.

9. Weber, K.T.; Brilla, C.G.; Janicki, J.S. Myocardial fibrosis. Functional significance and regulatory factors. Cardiovasc. Res. 1993, 27, 13-31.

10. Assyang, P.; Carré, F.; Chevalier, B.; Delcayre, C.; Mansier, P.; Swynghedauw, B. Compensated cardiac hypertrophy: Arrhythmogenicity and the new myocardial phenotype I. Fibrosis. Cardiovasc. Res. 1997, 34, 439-444.

11. Banerjee, I.; Yekkala, K.; Borg, T.K.; Baudino, T.A. Dynamic interactions between myocytes, fibroblasts, and extracellular matrix. Ann. N. Y. Acad. Sci. 2006, 1080, 76-84.

12. Alves-Pereira, M.; Castelo Branco, N.A.A. Vibroacoustic disease: Biological effects of infrasound and low-frequency noise explained by mechanotransduction cellular signaling. Prog. Biophys. Mol. Biol. 2007, 93, 256-279.

13. Diez, J. Mechanisms of cardiac fibrosis in hypertension. J. Clin. Hypertens. 2007, 9, 546-550.

14. Rakusan, K.; Wicker, P. Morphometry of the small arteries and arterioles in the rat heart: Effects of chronic hypertension and exercise. Cardiovasc. Res. 1990, 24, 278-284.

15. Chen, Y.; Torry, R.J.; Baumbach, G.L.; Tomanek, R.J. Proportional arteriolar growth accompanies cardiac hypertrophy induced by volume overload. Am. J. Physiol. 1994, 267, 2132-2137.

16. Khazaei, M.; Fallahzadeh, A.R.; Sharifi, M.R.; Afsharmoghaddam, N.; Javanmard, S.H.; Salehi, E. Effects of diabetes on myocardial capillary density and serum angiogenesis biomarkers in male rats. Clinics 2011, 66, 1419-1424.

17. Komaru, T.; Kanatsuka, H.; Shirato, K. Coronary microcirculation: Physiology and pharmacology. Pharmacol. Ther. 2000, 86, 217-261.

18. Amann, K.; Neususs, R.; Ritz, E.; Irzyniec, T.; Wiest, G.; Mall, G. Changes of vascular architecture independent of blood pressure in experimental uremia. Am. J. Hypertens. 1995, 8, 409-417.

19. Amann, K.; Wiest, G.; Zimmer, G.; Gretz, N.; Ritz, E.; Mall, G. Reduced capillary density in the myocardium of uremic rats-A stereological study. Kidney Int. 1992, 42, 1079-1085.

20. Mall, G.; Rambausek, M.; Neumeister, A.; Kollmar, S.; Vetterlein, F.; Ritz, E. Myocardial interstitial fibrosis in experimental uremia-Implications for cardiac compliance. Kidney Int. 1988, 33, 804-811.

21. Amann, K.; Ritz, E. Reduced cardiac ischaemia tolerance in uraemia-What is the role of structural abnormalities of the heart? Nephrol. Dial. Transplant. 1996, 11, 1238-1241.

(C) 2015 by the authors; licensee MDPI, Basel, Switzerland. This article is an open access article distributed under the terms and conditions of the Creative Commons Attribution license (http://creativecommons.org/licenses/by/4.0/). 